\title{
Defect detection in fiberglass reinforced epoxi composite pipes reproducing field inspection conditions
}

\author{
by Souza M. P. V', Rebello J. M. A ${ }^{1}$, Soares S. D², Freitas G. A. $C^{2}$ \\ ${ }^{1}$ Federal University of Rio de Janeiro - Metallurgy and Materials Engineering Dep. \\ ${ }^{2}$ PETROBRAS - Brazilian Petroleum
}

\begin{abstract}
Many works that adopt active thermography as a non-destructive inspection method use very sophisticated equipment for test piece thermal excitation and data acquisition, which demand highly prepared operators, causing their application in industrial environments very costly. This paper aims at detecting defects in adhesive joints of fiberglass reinforced epoxi pipes by means of the Fourier transform phase angle analysis of the time evolution pixel amplitude, using simple and low-cost equipment. Results show that is possible to detect defects in adhesive joints, studying the phase angle behavior, even in regions, where the thermograms show no defects.
\end{abstract}

\section{Introduction}

Infrared thermography is a well known technique that provides the capability to see the unseen. As a nondestructive method of inspection, it can reveal surface, subsurface and internal structural defects over a wide variety of materials. The technique is based on the reflectiveness of thermal waves by the surface of the materials $[1,2]$.

The thermographic technique is showing a good performance on the inspection of composite materials, including adhesive layers $[3,4]$ and has been used by numerous sectors of the industry reaching exciting levels of detection and characterization of a large variety of materials and devices, justifying the great number of studies that have been made recently [4].

The active thermography is the kind of infrared inspection that uses the heat of external sources as excitation that can be analysed on permanent or transient state of heat flow. The lockin thermography uses the information of a unique frequency to analyse the amplitude and phase angles relating these data to location and size of the defects. The pulsed thermography analyses the decay on time of a pulse heat excitation without selecting a specific frequency $[1,2]$.

The Pulsed Phase Thermography is an active method that allows the analysis of a wide band of frequencies of heat excitation giving information about the magnitude and phase angles of the temperature decay of the objects inspectioned. The behavior of the phase angle can indicate the presence of defects even inside the material's structure [1,2], not detectable by conventional termography.

\section{Pulsed Phase Thermography}

Pulsed phase thermography allows the analysis of a series of frequencies by means of a single excitation, and it can be performed in a fast way [2]. However, the equipment used in all active techniques still represents some difficulty for a fast and reliable application in the industry environment, because of the high level of qualification necessary for the operators to set and use the equipment, as well as the high costs involved.

The information that can be extracted from the pulsed thermography pass through math algorithms to be analyzed on a frequency basis less affected by the noise of the thermal excitation allowing the observation of phase angles over a wide spectrum of frequencies and can be used to compose the phase images to visualize defects non-detectable on the original thermograms $[1,5]$.

\subsection{Phase Angle Analysis}

Phase angle analysis is a well known technique, with a great potential in the area of thermography [3]; being a kind of datum that is not difficult to obtain, it is less influenced by thermal excitation anisotropy and it allows measuring the depth of the defects, as the phase carries information about the thermal wave path $[1,2,5]$. The lock-in thermography can analyze, for a single frequency, the information contained in the infrared image, but it is not a technique easy to be repeated.

The process known as pulsed phase thermography uses the easy to aplicate experimental method of the pulsed thermography and extracts the phase angle information from the temperature decay right after the excitation of a heat source. Its main advantages are the analysis of multiple frequencies with a unique heat excitation, easy repeatability and more simple equipment than in other methods.

The phase angle information is extracted perfoming the fast Fourier transform (FFT) of the temperature decay during time after an excitation. Defective regions present relevant differences on the phase behavior related to non-defective regions $[5,6]$. 


\section{Methodology}

In this work, the method used was heating the test piece and then recording the temperature decay curve in the transient state of heat flow. Heating was performed in reflection using a hot air industrial blower and a high power halogen lamp. The only controlled parameters were excitation time and the distance from the exciter to the test piece (Fig 1).

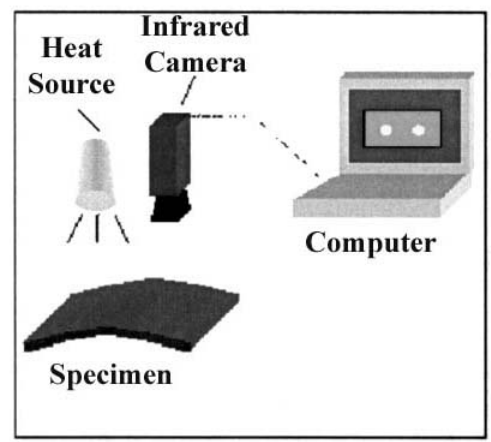

Fig 1. Scheme of heating and recording method

A Flir Prism Single Point infrared sensor was used in order to acquire the infrared images series. The test pieces were $60 \times 80 \mathrm{~mm}$ and 6" pipe sections with $4 \mathrm{~mm}$ thickness fiberglass reinforced epoxi containing circumferential defects with 5 to $10 \mathrm{~mm}$ of diameter and varied depths divided into two classes: lack of adhesive and holes (lack of material), shown in Fig. 2. The heat excitation time was 5 s and the distance of the exciter and the infrared camera to the test pieces was $600 \mathrm{~mm}$.

(a)
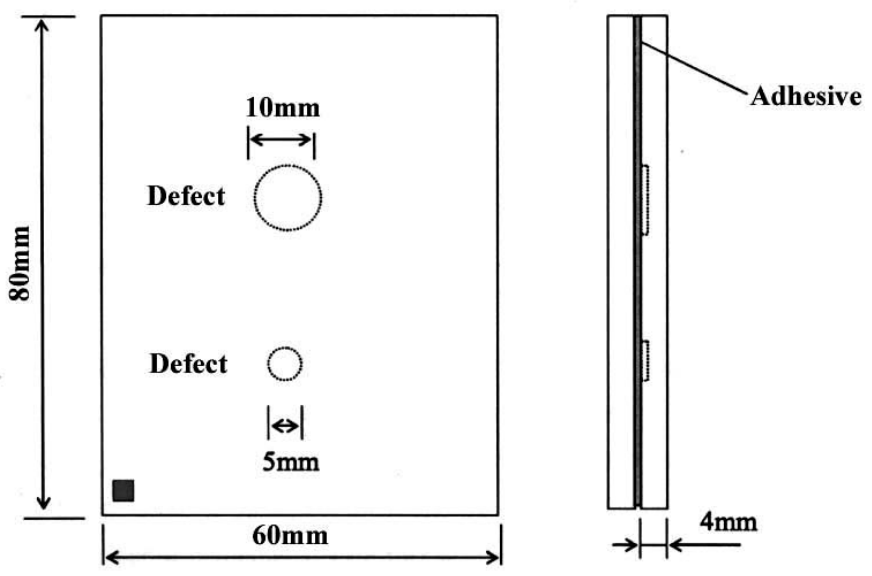

(b)
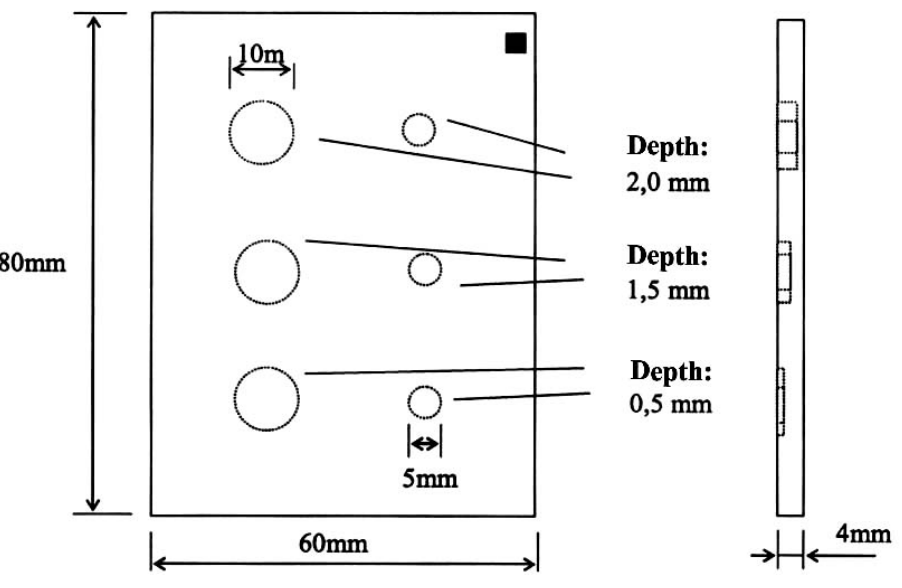

Fig 2. Specimen inspectioned: (a) lack of adhesive (b) holes

A series of infrared images was recorded and a function corresponding to the time evolution of the thermal wave at pixel level was obtained during the cooling of the specimen. Then, it was possible to obtain the fast 


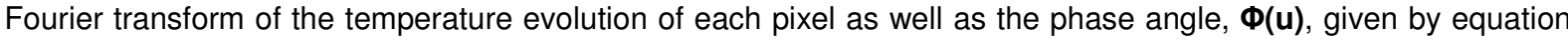
1 , using a MatLab routine developed for this task (Fig. 3 ).

$$
\Phi(\mathrm{u})=\tan ^{-1}[\operatorname{lm}(F(\mathrm{f})) / \operatorname{Re}(F(\mathrm{f}))]
$$

where $\operatorname{Im}(F(\mathrm{f}))$ and $\operatorname{Re}(F(\mathrm{f}))$ are the imaginary and real parts of the Fourier transform

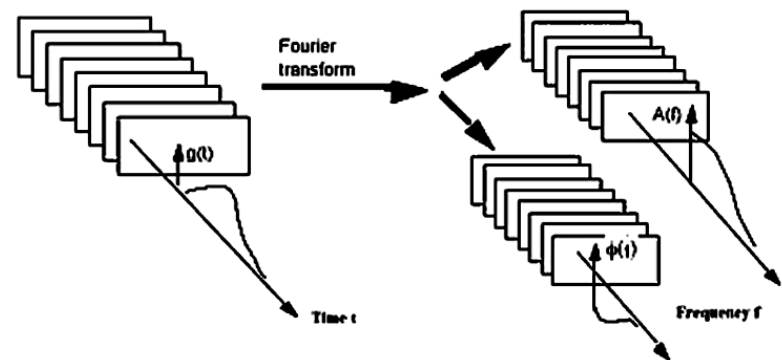

Fig 3. Amplitude and phase extracted from temperature evolution by FFT [7].

The evolution of the phase angle in the frequency domain of several pixels of defective regions were compared with a pixel of a non-defective region, which was used as a reference through the difference between the values of the defect and the reference phase angles. Contrast profiles were also measured in order to visualize, in the thermograms, the visible and non-visible defective regions.

(a)

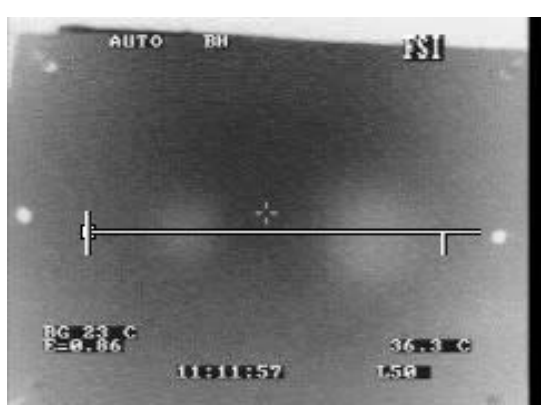

(b)
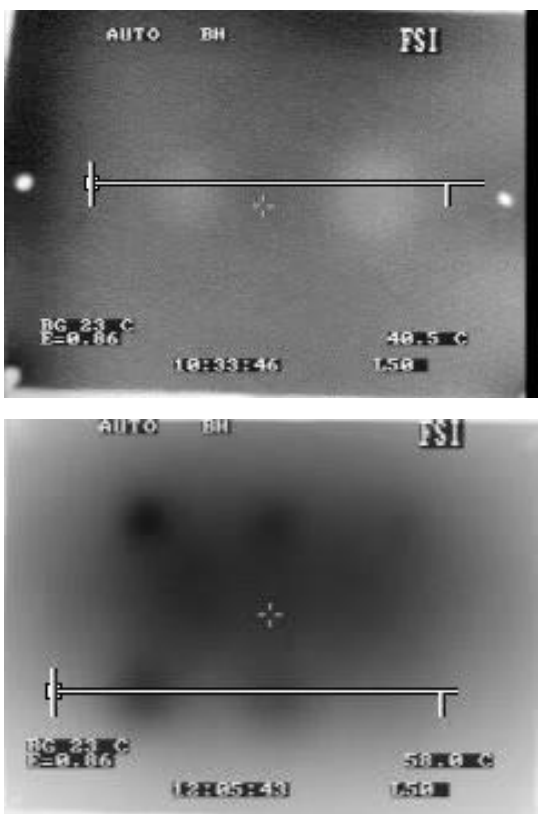
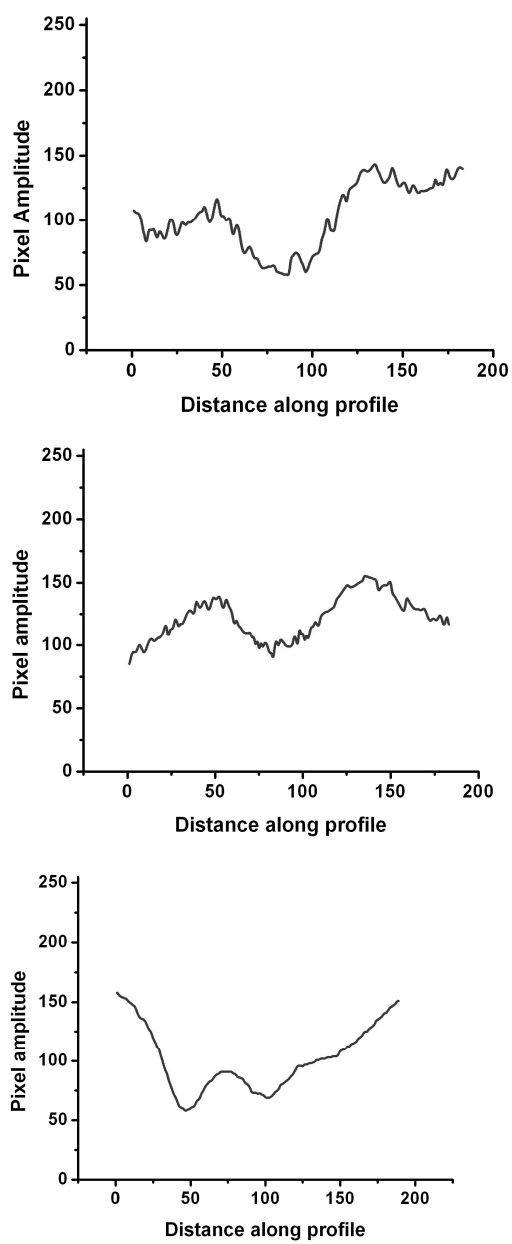

Fig 4. Constrast Profiles: (a) Specimen 1 - halogen lamp excitation; (b) Specimen 1 - air blower excitation; (c) Specimen 2 - halogen lamp excitation 


\section{Results and Discussion}

Some contrast profiles were measured in the best frames of the infrared videos of the two specimen inspectioned (Fig 4).On specimen 1, the profiles can detect all the defects for both methods of excitation. Although, for specimen 2, one defective region wasn't detected by the contrast profile (Fig 4-c).

The phase angle analysis was then implemented in order to be compared with the profiles by the difference of phase angle behavior trying to confirm the defects detected previously and, much more important, detect the defects that were not revealed by the contrast profiles.

There was a clear difference in the behavior of the phase angle variation between defective and intact regions, mainly at low frequencies. Figures 4,5 and 6 show the phase angle difference graphics versus the frequency of two specimen heated with the halogen lamp and air blower, showing the difference of behavior among phases for non-defective, visibly defective, little visibly defective, and invisible-to-the-naked-eye defective regions, in the infrared images.

Figures 5-a, 6-a and 7-a show the difference of phase angles between two non-defective regions. The variation of phase angle was near to zero all along the graph. Figures 5-b, 6-b and 7-b show the behavior of the phase angle difference between a non-defective region and a defective region easily detectable with the naked eye and the contrast profile, confirming the noticeable deviation caused by a defect on the phase angle behavior. Figures 5-c and 6-c show the phase angle difference between a non-defective region and another that is difficult to detect on naked eye inspection and show little deviation on the contrast profile.

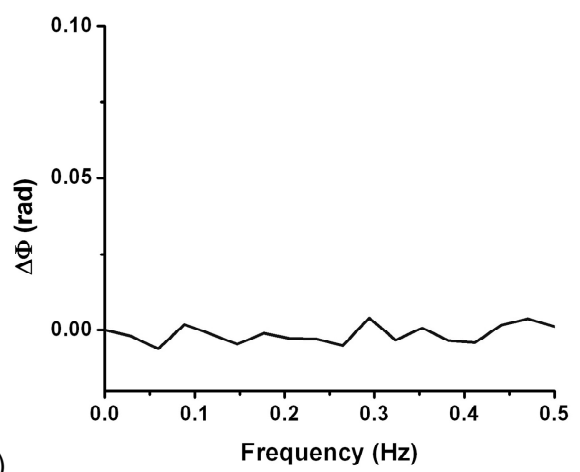

(a)

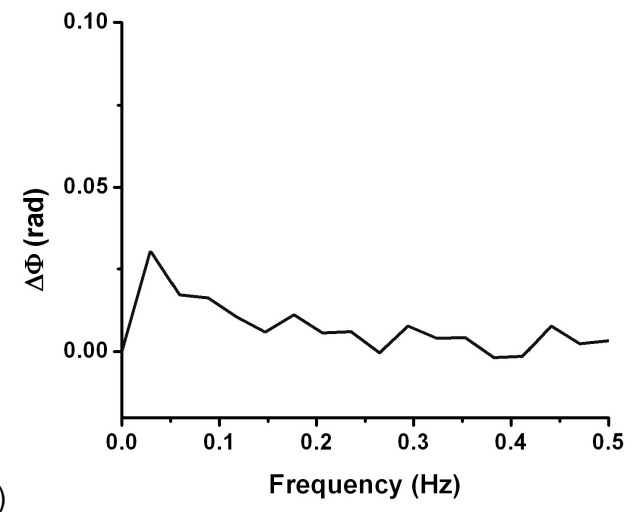

(c)

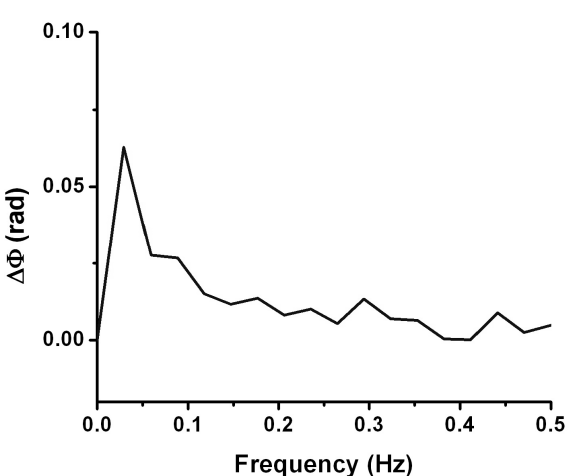

(b)

Fig 5. Difference of phase angles on specimen 1 using halogen lamp excitation: (a) non-defective region (b) region with $2,0 \mathrm{~mm}$ of depth defect visible in the thermogram (c) region with $1,5 \mathrm{~mm}$ of depth defect little visible in the thermogram 


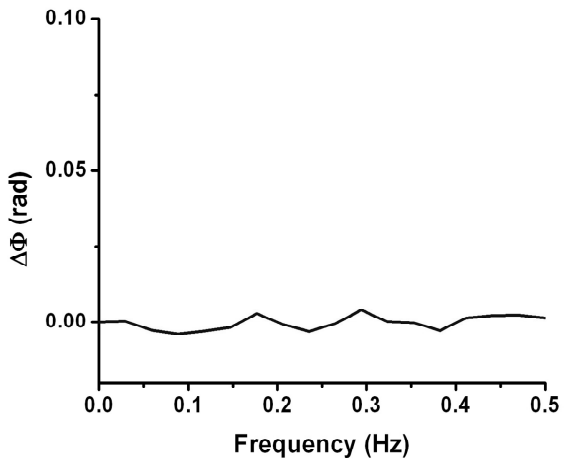

(a)

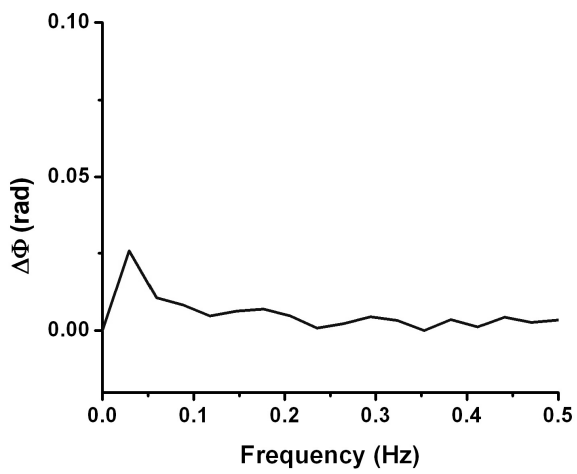

(c)

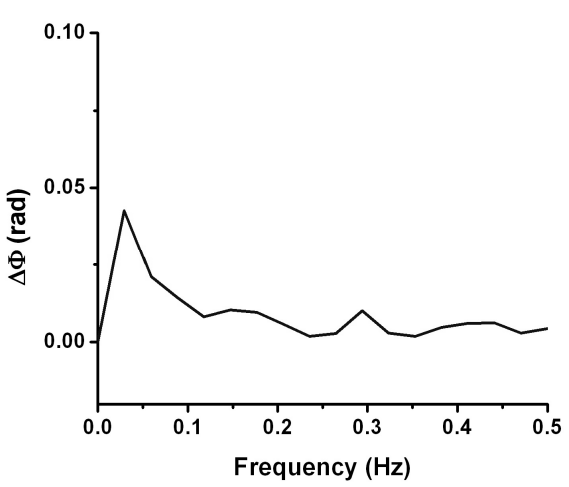

(b)

Fig 6. Difference of phase angles on specimen 1 using air blower excitation: (a) non-defective region (b) region with 2,0 mm of depth defect visible in the thermogram (c) region with 1,5 $\mathrm{mm}$ of depth defect little visible in the thermogram

In order to confirm the potentiality of the technique, defective regions that were not visible to the naked eye in any of the infrared images of the video were compared with the same previous reference (Figure 7-c); and, despite of the low amplitude, the difference in the behavior was once more noticed.

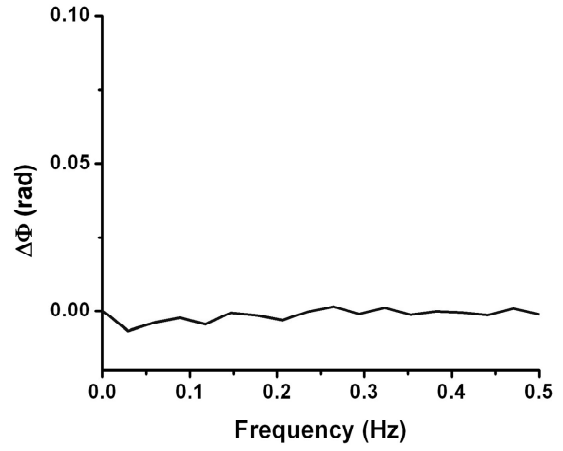

(a)

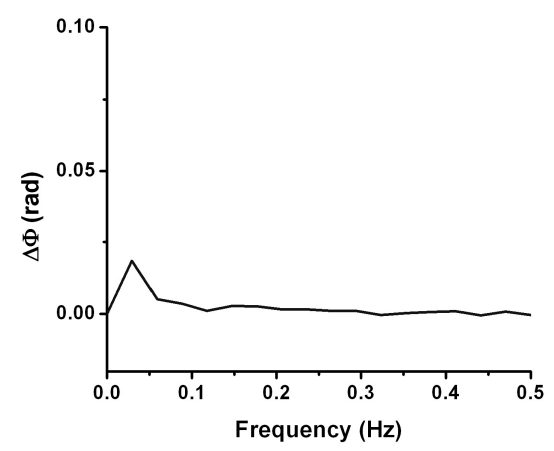

(c)

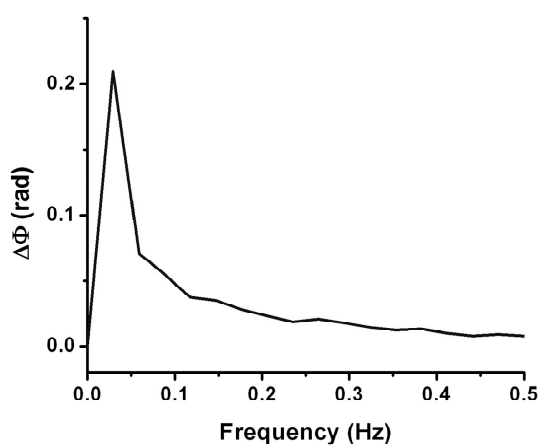

(b)

Fig 7. Difference of phase angles on specimen 2 using halogen lamp excitation: (a) non-defective region b) region with $2,0 \mathrm{~mm}$ of depth defect visible in the thermogram (c) region with $0,5 \mathrm{~mm}$ of depth defect non-visible in the thermogram 
Gonzalez [5] extracted absolute phase contrast graphs of each pixel in thermograms using photographic flash as heat excitation and hough transform to construct the blind frequency images of Plexiglas composite specimen.

Some works obtained phase angle information and phase images on composite and polymer materials thermograms achieving good results but using very sophisticated methods and equipments [5-10] that could not fit field inspections in industrial environments.

The results shown in this work are innovative based on the simplicity of the method and low cost of equipments used, stimulating more studies intending to achieve an industrial applicable active thermographic method of inspection.

\section{Conclusions}

The phase angle analysis by the Fourier transform of time evolution pixel amplitude can detect holes (lack of material) and lack of adhesive of fiberglass reinforced epoxi pipes structure and adhesive joints comparing the phase angle behavior between defective and non-defective areas of the thermograms in field inspection conditions.

The preliminary results show the potentiality of the technique in the detection of typical composite pipe defects and support the realization of more tests aimed at the generalization of the results for other composite materials, excitation forms and types of defects.

The use of low cost equipment and simple mathematical concepts attempts to the great capability to implementation of the technique on industrial environments.

These results can also be used to establish a frequency for the composition of the phase images in order to precisely locate the little visible and invisible defects in the infrared images.

\section{REFERENCES}

[1] X. Maldague, Theory and Practice of Infrared Technology for Nondestructive Testing, Wiley-Interscience (2001).

[2] X. Maldague, P. Moore, Nondestructive Testing Handbook - Infrared and Thermal Testing, Volume 3, American Society for Nondestructive Testing.

[3] J. A. Schroeder, T. Ahmed, B. Chaudhry, S. Shepard, Non-destructive testing of structural composites and adhesively bonded composite joints: pulsed thermography, Composites: Part A 33 (2002), 1511-1517.

[4] X. E. Gros, Current and Future Trends in Non-destructive Testing of Composite Materials, Annales de Chimie Science des Matériaux, 25, Issue 7 (2000) 539-544.

[5] D. A. González, C. Ibarra-Castanedo, F. J. Madruga, X. Maldague, Differentiated absolute phase contrast algorithm for the analysis of pulsed thermographic sequences, Infrared Physics \& Technology 48 (2006) 16-21.

[6] H. Gerhard, G. Busse, Lockin-ESPI interferometric imaging for remote non-destructive testing, NDT\&E International, 39 (2006) 627-635.

[7] François Galmiche, Xavier Maldague, Pulsed Phase and Thermal Tomographie: A Comparison, PACNDT 98 - Proceedings.

[8] C. Meola, G. M. Carlomagno, A. Squillace, A. Vitiello, Non-destructive evaluation of aerospace materials with lock-in thermography, Engineering Failure Analysis 13 (2006) 380-388.

[9] T. Zweschper, A. Dillenz, G. Riegert, D. Scherling, G. Busse, Ultrasound excited thermography using frequency modulated elastic waves, Insight 45, Issue 3 (2003) 178-182.

[10] J. Rantala, D. Wu, G. Busse, NDT of polymer materials using lock-in thermography with water-coupled ultrasonic excitation, NDT\&E International 31 (1997) 43-49 